\title{
Measurements from The Heavy-Ion Telescope at The ISS Kibo Exposed Facility
}

\author{
By Haruka Ueno, ${ }^{1)}$ Haruhisa Matsumoto, ${ }^{1)}$ Kiyokazu KogA ${ }^{1)}$ and Aiko Nagamatsu ${ }^{2)}$ \\ ${ }^{1)}$ Aerospace Research and Development Directorate, JAXA, Tsukuba, Japan \\ ${ }^{2)}$ Human Spaceflight Technology Directorate, JAXA, Tsukuba, Japan \\ (Received July 31st, 2015)
}

Space radiation such as solar energetic particles, galactic cosmic rays (GCR), and trapped particles affect space activities. Heavy ions, in particular, have high linear energy transfer (LET) that exacerbates the risk of radiation exposure for astronauts and affects the electrical circuits of satellites. The Japan Aerospace Exploration Agency (JAXA) has operated the Space Environment Data Acquisition Equipment - Attached Payload (SEDA-AP) at the International Space Station (ISS) Japanese Experiment Module (Kibo) - Exposed Facility, since 2009. The heavy-ion telescope (HIT) is one of the SEDA-AP instruments, which comprises two positionsensitive silicon detectors and 16 silicon detectors. Based on the $\Delta E \times E$ particle-identification method, HIT measures fluxes of energetic ions from $\mathrm{Li}$ to $\mathrm{Fe}$ and calculates the loss of energies at each sensor. The HIT results are consistent with the general GCR model and other ISS experiments in terms of elemental abundances and LET distributions. In addition, HIT might have detected heavy ions from a X5.4 solar flare. Moreover, there is the possibility of detecting the anomalous cosmic rays (ACR) from the ADEOS satellite observations; however, ACR was not observed because the pitch angle was not in the field of view of HIT or no distribution of ACR exists at $400 \mathrm{~km}$ altitude.

Key Words: Silicon Detector Telescope, Heavy Ions, Kibo Exposed Facility, SEDA-AP

Nomenclature

$\begin{array}{lll}E & : & \text { energy } \\ M & : & \text { mass of particle } \\ n & : & \text { number of SSD } \\ x & : & \text { track length } \\ z & : & \text { atomic number }\end{array}$

\section{Introduction}

Space radiation is mainly categorized as solar energetic particles (SEP), trapped particles, and galactic cosmic rays (GCR). Other examples of solar radiation are the low-energy solar wind and anomalous cosmic rays (ACR). ${ }^{1)}$ The energy distribution of the various space radiation examples is shown in Fig. 1. Space activities are affected by space radiation. For example, space radiation results in electronic circuit errors and electrifications for satellite or the international space station (ISS) as well as the radiation exposure for astronauts. In particular, although such fluxes are low, the heavy ions from SEP and GCR are particles with high linear energy transfer (LET), and thus, they strongly affect electronic circuits or human cells. This makes having data on heavy ions necessary.

\section{Overview of SEDA-AP and HIT}

\subsection{SEDA-AP}

Since 2009, JAXA has operated the Space Environment Data Acquisition Equipment - Attached Payload (SEDA-AP) to collect data for space radiation and other hazards at the ISS Kibo Exposed Facility (EF). ${ }^{2)}$ The position of SEDA-AP is shown in Fig. 2. Besides collecting space environment data, SEDA-AP builds databases for the ISS orbit environment. Also the first experiment at EF was to verify the on-orbit attached payload bus technology. SEDA-AP uses seven sensors to collect space environment data. The equipment and location of each sensor

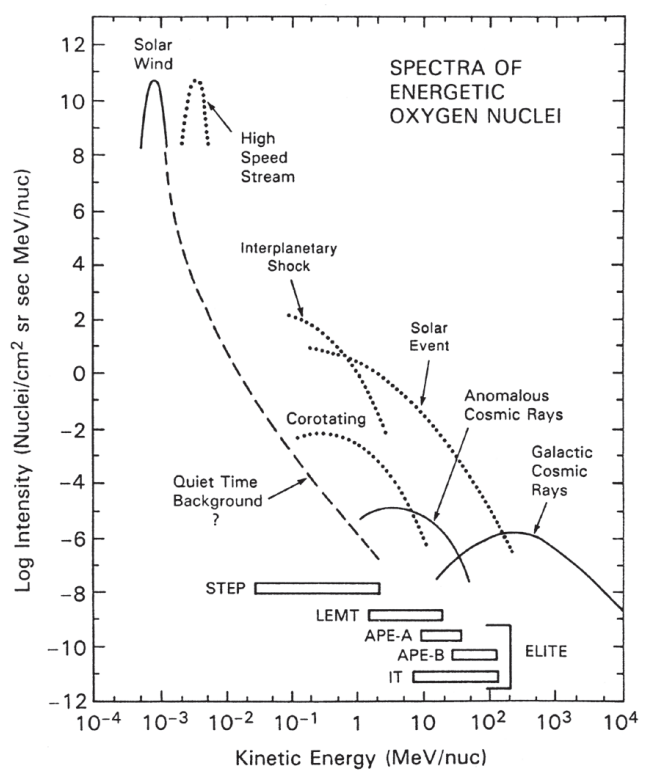

Fig. 1. Typical oxygen spectra for different populations of interplanetary particles taken from the EPACT investigation.

Table 1. SEDA-AP specifications

\begin{tabular}{|l|l|}
\hline Dimensions $(\mathrm{W} \times \mathrm{H} \times \mathrm{L})$ & $0.8 \mathrm{~m} \times 1.0 \mathrm{~m} \times 3.0 \mathrm{~m}$ \\
\hline Mass & ca. $450 \mathrm{~kg}$ \\
\hline Power Consumption & ca. $220 \mathrm{~W}$ (nominal) \\
\hline
\end{tabular}

are shown in Fig. 3. Table 1 lists the SEDA-AP specifications. When the mast is extended, the size is $0.8 \mathrm{~m} \times 1.0 \mathrm{~m} \times 3.0 \mathrm{~m}$. SEDA-AP will enter the extended operation in autumn of 2015. Port numbers will be changed from \#9 to \#11 in the extended operation.

\subsection{HIT}

The heavy-ion telescope (HIT), which is one of the sensors on SEDA-AP, measures the flux of energetic ions from $\mathrm{Li}$ to $\mathrm{Fe}$ based on the $\Delta E \times E$ method $^{3)}$ to identify nuclear species. The 


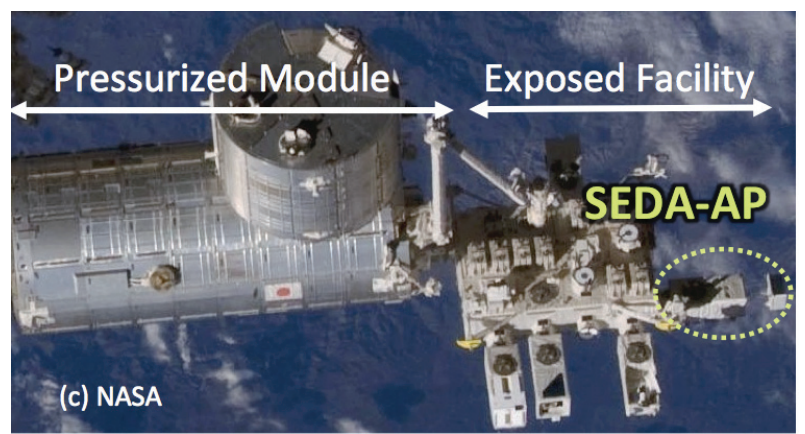

Fig. 2. Position of SEDA-AP in EF.

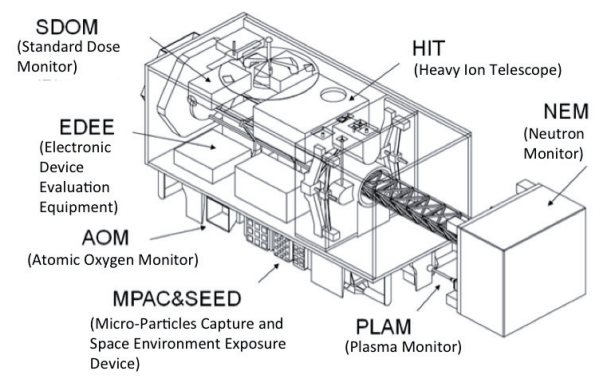

Fig. 3. Positions of the seven sensors in SEDA-AP.

Bethe-Bloch equation describes the loss of energy for charged particles deposited on materials. When the velocity of particles is low, it is approximated by Eq. 1:

$$
-\frac{d E}{d x} \propto \frac{M z^{2}}{E}
$$

where $E, x, M$ and $z$ are the energy, track length, mass of particle, and atomic number, respectively. The value of $\Delta E \times E$ is unique to the atomic number; thus, incident particles are identified by using this relation.

A schematic of HIT is shown in Fig. 4. HIT comprises two position-sensitive silicon detectors (PSD1 and PSD2) and 16 PIN-type silicon detectors (SSD1-SSD16). SSD16 can be used as a veto sensor. Two $50 \mu$ m-thick aluminum shielding films are placed at the top of HIT to reject electrons and low-energy particles. HIT specifications are summarized in Table 2. The energy ranges are simulated by using the PHITS code, ${ }^{4)}$ which is a general-purpose Monte Carlo particle-transport simulation code.

An incident particle creates an electrical pulse that is proportional to the value of the energy loss in the silicon detectors. HIT uses the coincidence between two PSDs with the particle discrimination level as trigger. When coincidence is detected, a charge-sensitive preamplifier reads the signals from each sensor and sends them to a shaping amplifier. If a particle hits the silicon sensors, the incident energy can be measured and the atomic number can be identified using the $\Delta E \times E$ method. HK data are sent as data packets every $4 \mathrm{~s}$ when HIT is in a lowincidence particle area.

\section{Observation}

\subsection{The changes of $\mathrm{DL}$}

HIT uses the coincidence between two PSDs with the discrimination level (DL) as a trigger. Four grades of DL for PSD1 and PSD2 were used to prevent saturation caused by protons

\begin{tabular}{|l|l|}
\multicolumn{2}{c}{ Table 2. } \\
\hline Sensors & PSD1: $200 \mu \mathrm{m}, 62 \times 62 \mathrm{~mm}^{2}$ \\
& PSD2: $400 \mu \mathrm{m}, 62 \times 62 \mathrm{~mm}^{2}$ \\
& SSD1-16: $420 \mu \mathrm{m}, 85 \mathrm{~mm}^{2} \phi$ \\
\hline Shielding film & Al: $50 \mu \mathrm{m}+50 \mu \mathrm{m}$ \\
\hline G-factor & $25 \mathrm{~cm}^{2} \mathrm{str}$ \\
\hline Energy range & Li: $10-43 \mathrm{MeV} / \mathrm{n}$ \\
& C: $16-68 \mathrm{MeV} / \mathrm{n}$ \\
& O: $18-81 \mathrm{MeV} / \mathrm{n}$ \\
& Si: $25-111 \mathrm{MeV} / \mathrm{n}$ \\
& Fe: $34-152 \mathrm{MeV} / \mathrm{n}$ \\
\hline Time resolution & $0.1 \mathrm{sec} /$ event \\
\hline Operation temperature & $-30-+75 \mathrm{deg} .($ Elec. $)$ \\
& $-55-+40 \mathrm{deg} .($ Sensor $)$ \\
\hline Dimension & $52 \mathrm{~cm} \times 42 \mathrm{~cm} \times 23 \mathrm{~cm}$ \\
\hline Mass & $25 \mathrm{~kg}$ \\
\hline Power dissipation & $36 \mathrm{~W}$ \\
\hline
\end{tabular}

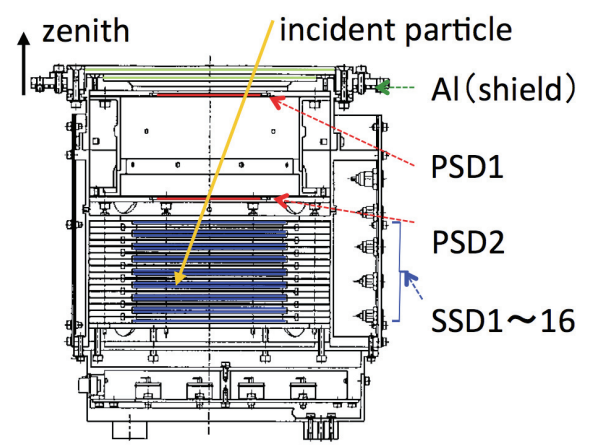

Fig. 4. Schematic showing HIT components. HIT detects the incident ions coming from the zenith direction.

during data processing. Figure 5 represents the relation between energy of incident particles of each element and DL in PSD1. The detectable particles at DL1, DL2, DL3, and DL4 were greater than protons, $\mathrm{He}, \mathrm{Li}$, and $\mathrm{C}$, respectively. The operational DL was changed three times during the entire observation period in 2010, 2012, and 2014. The correspondence between DL and observation period is given in Table 3.

\subsection{Fluctuations of other parameters}

Figure 6 shows the ISS altitude during the entire observation period. Affected by air resistance, the ISS altitude constantly decreases 200 m per day. In addition that ISS made a Reboost by own thruster, Automated Transfer Vehicle (ATV2) was used on July 2011 during docking to increase the altitude from about $350 \mathrm{~km}$ to $400 \mathrm{~km}$. On average, the ISS altitude is about 400 $\mathrm{km}$.

Monitoring solar activity is necessary because the flux of GCRs and solar activity, represented by the number of sunspot and solar flares, are negatively correlated. Solar events tend to occur at solar maximum and are associated with heavy ions. Figure 7 shows that during the observation period, the solar activity changed from minimum to maximum and that HIT observations were made almost at solar maximum. The data source of sunspot number is taken from WDC-SILSO, Royal Observatory of Belgium, Brussels.

\subsection{Operation rates}

Figure 8 shows the count rates from 08/06/2009 to $01 / 01 / 2015$. Owing to the high-beta angle, the sensors often 
Table 3. Discrimination level of each period.

\begin{tabular}{|l|l|l|l|l|}
\hline $\begin{array}{l}\text { Start- } \\
\text { End }\end{array}$ & $2009.8-$ & $2010.7-$ & $2012.8-$ & $\begin{array}{l}2014.7- \\
\text { present }\end{array}$ \\
\hline DL & 1 & 3 & 2 & 1 \\
\hline
\end{tabular}

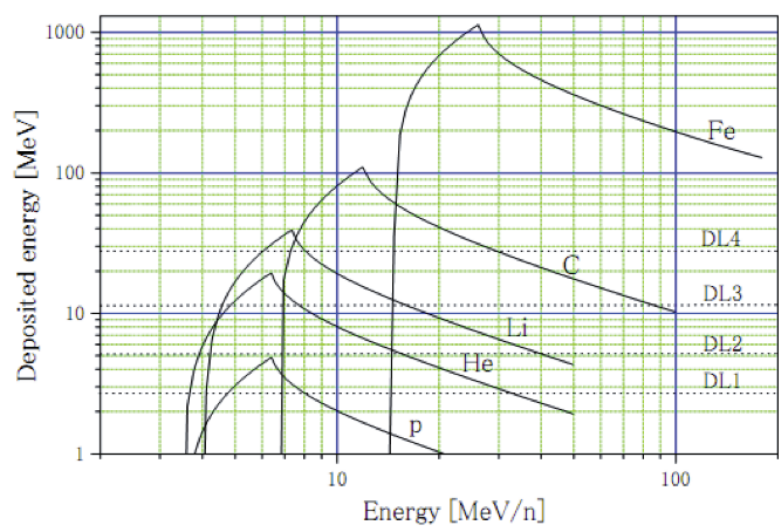

Fig. 5. Relation between incident particles and DL in PSD1. Higher DL can be used to observe the higher atomic number particles.

exceeded the temperature limits stopping the operations. Thus, the net HIT observation time was 1014 days and the rate of operation was approximately $51 \%$. The mean count rates of each DL are $2.10 \times 10^{-2}(\mathrm{DL} 1), 5.39 \times 10^{-3}(\mathrm{DL} 2)$ and $8.96 \times 10^{-4}$ (DL3).

\section{Data analysis}

\subsection{Particle identification}

HIT recorded 760, 320 events during five and a half years. The data were processed with a ground-based software. To precisely calculate the energy loss, the coefficients of the distorted correction of PSD and the engineering conversion values acquired from calibration data were used in data processing. The incident angle from PSDs is used to correct the energy loss that depends on the track length. We set $\Delta E(\equiv d E / d x$ in Eq. 1) and the total energy for particle identification below. As seen in Fig. 9 , when a incident particle stops at $\operatorname{SSD} n(1 \leqslant n \leqslant 16), \Delta E$ is the energy loss in the $\operatorname{SSD}(n-3)$. The total energy (TE) is the sum of the energy loss in $\operatorname{SSD} n, \operatorname{SSD}(n-1)$ and $\operatorname{SSD}(n-2)$. Using $\Delta E$ and total energy, the incident particles are identified after noise elimination. Atomic numbers are identified by comparing measured and theoretical $\Delta E \times E$ values.

After this data processing, events that penetrate SSD16 are about $17.6 \%$ of the times HIT has triggered. The number of events, which are identified both of $z$ and incident energy, is $1.4 \%(10,445)$. The non-identified $z$ are $54.4 \%$, which are caused by penetrating the sides of sensors as one of the reasons. Other particles $(26.6 \%)$ are identified $z$ but non-identified incident energy. Figure 10 shows the $\Delta E \times E$ plot in case that a particle stops in SSD2 for identified $z$ and incident energy. The dot lines are calculated values obtained by the simulation of loss energy of the particles to each SSD of each atomic number. Line bending corresponds to the case of penetration.

Figure 11 shows the geographic distribution of protons, heliums, carbons, and irons for the entire observation period. At high latitudes and the SAA region, the particles easily penetrate the geomagnetic field at low altitude because of the low cut-off

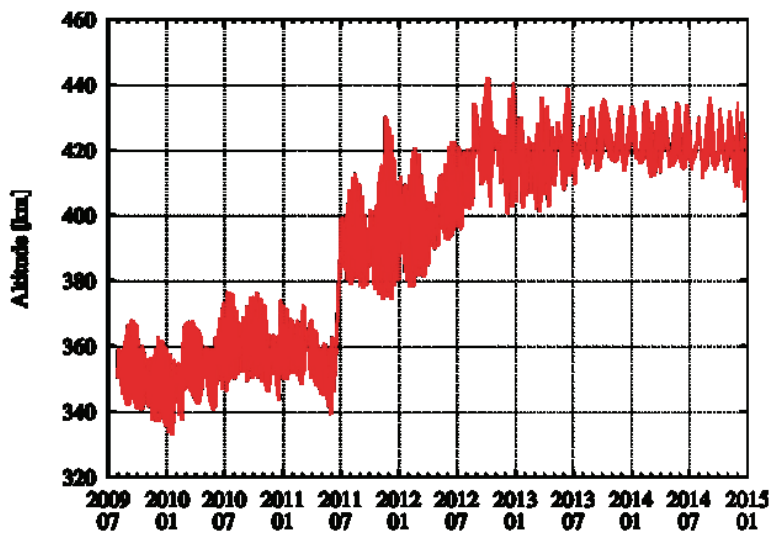

Fig. 6. ISS altitude during the observation period. ISS was reboosted by AVT2 on July 2011.

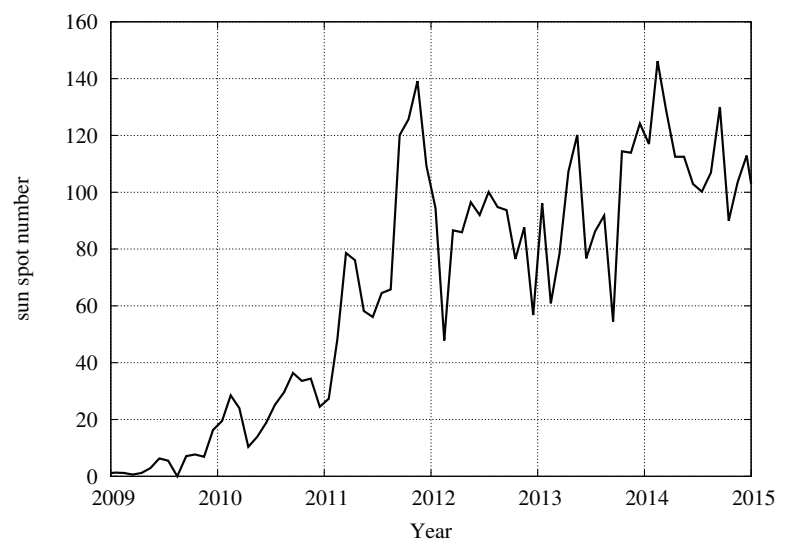

Fig. 7. Solar activity during the observation period. The number of sun spots and solar activity correlate. Solar activity was almost maximum in this period.

rigidity.

\subsection{Abundance ratio}

The abundance of He:Fe is shown in Fig. 12. Even nucleons seem to have higher abundance than odd nucleons. To evaluate the data, we compare the relative abundance of nucleons observed with HIT and calculated with the CREME96 ${ }^{5)-7)}$ model. The CREME96 model is used to calculate single-event upset rates in devices using GCR spectra. The CREME96 model and observed data for each DL period are shown in Fig. 12. The lower panel of Fig. 12 shows the ratio of the abundance ratio of CREME96 and HIT. HIT data is higher than CREME96 in the range of $z \leqslant 16$, especially DL3 data is higher than CREME96 in the range of $6 \leqslant z \leqslant 16$. HIT may have observed heavy ions from solar flares so that there is difference in the abundance ratio. A X5.4 class solar flare on March 2012 was considered responsible for the difference (see section 6).

\subsection{LET distributions}

Figure 13 shows the differential LET fluxes for PSD1 in each DL period for all events, including non-identified particles that passed through the detector. The LET were converted to the value of water. HIT observed several peaks caused by $\mathrm{O}, \mathrm{Ne}$, $\mathrm{Mg}, \mathrm{Si}$, and $\mathrm{Fe}$, which corresponded to LET peaks of 13, 20, $29,39,135 \mathrm{keV} / \mu \mathrm{m}$, respectively. The data revealed that the observed LET range changed depending on the DL. A comparison of LET flux data with the CREME96 model, using the 


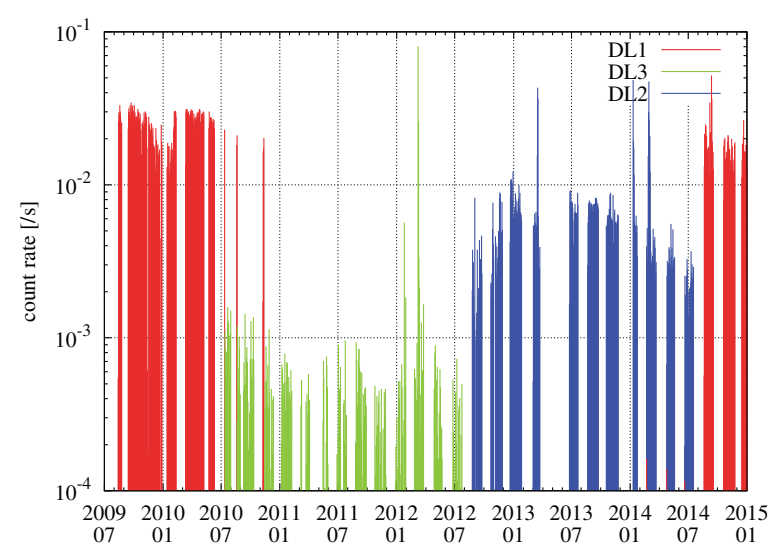

Fig. 8. Count rates during the entire observation period. The red, blue, and green lines are for the DL1, DL2, and DL3 data, respectively. The count rate flares and times correspond with solar flares.

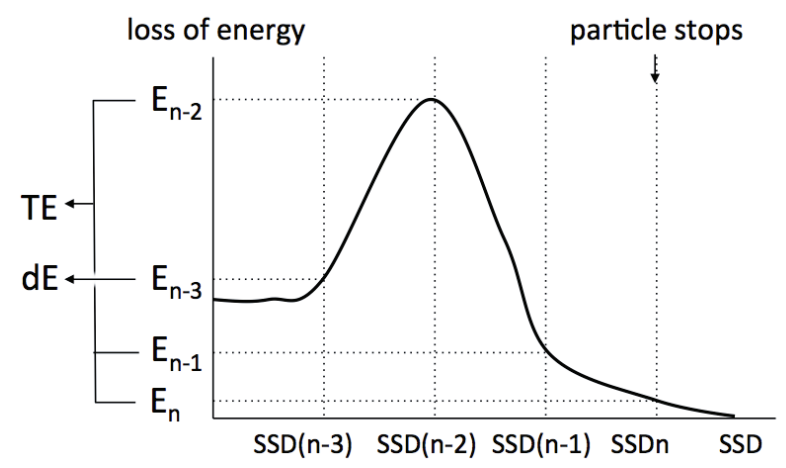

Fig. 9. Relation between the loss of energy of an incident particle in each SSD and the selection of TE and $\Delta E$

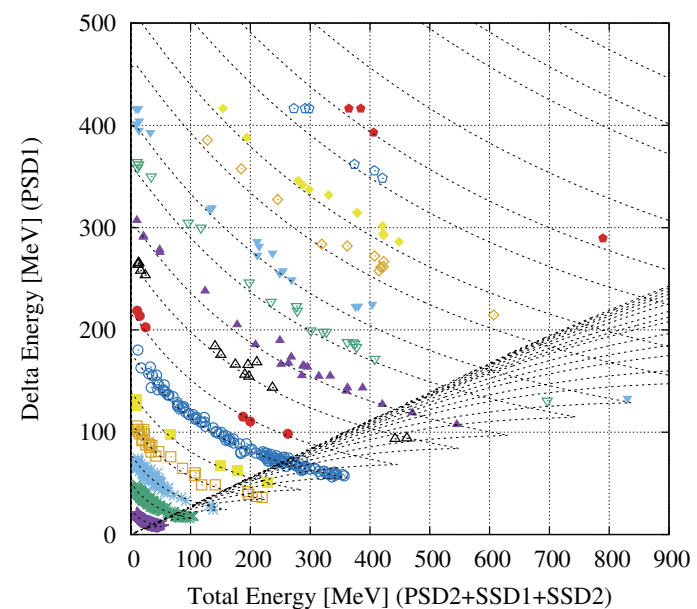

Fig. 10. $\Delta E \times E$ plot for identified particles in case that stop detector is SSD2. Dot lines are calculated values.

same initial conditions described in section 4.1, is shown in Figure 11. Although LET from HIT an order of magnitude greater than that of CREME model over $200 \mathrm{keV} / \mu \mathrm{m}$, HIT data is approximately corresponds with the model.

The LET flux in the DL3 period was greater than that in the other periods above $200 \mathrm{keV} / \mu \mathrm{m}$. This suggests that many particles with energy distributions that differed from GCR were intercepted by HIT during this period. As mentioned in section 4.2, the X5.4 flare, which occurred in 2012, also affected the LET flux distributions. This is discussed in detail in section 6 .
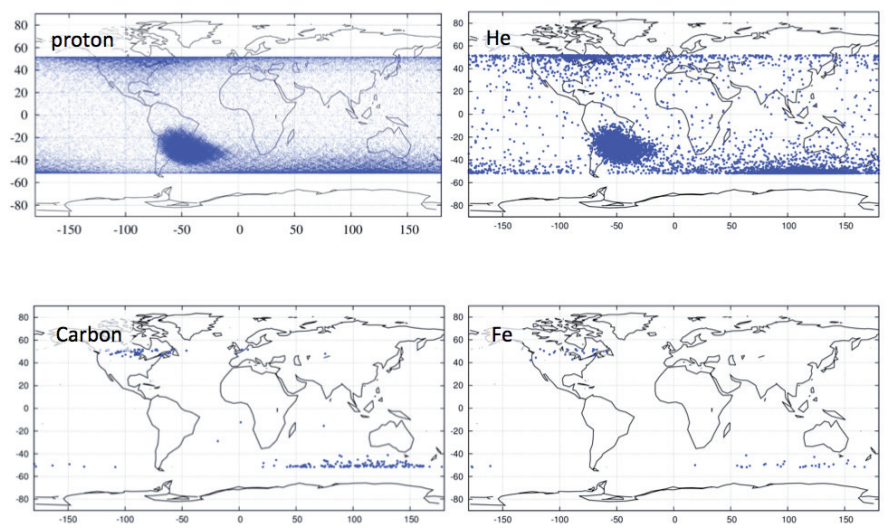

Fig. 11. Geographic distribution of proton, $\mathrm{He}, \mathrm{C}$ and $\mathrm{Fe}$ in whole observation. The main distributions are seen at high latitude for all ions and at SAA region for proton, He.

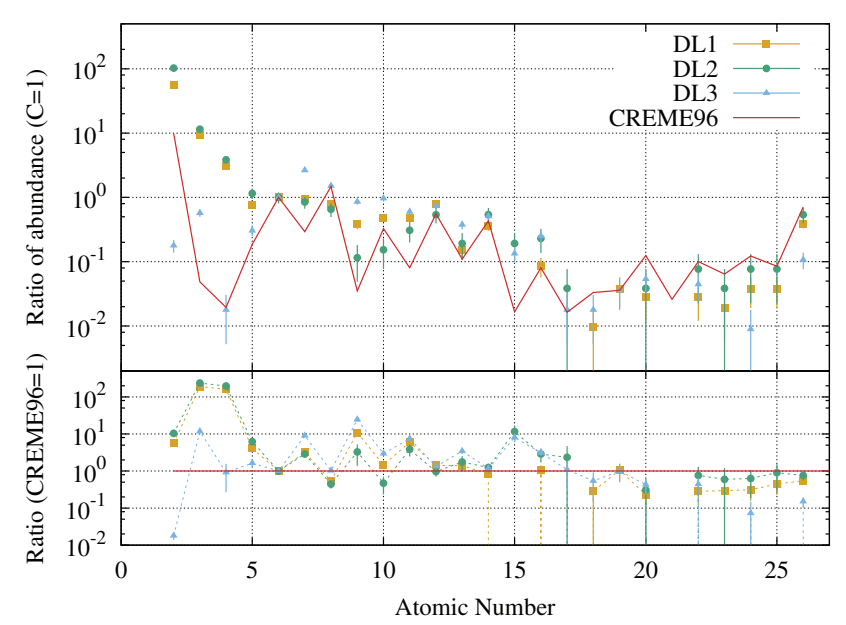

Fig. 12. upper panel: Elemental abundances in DL 1 (square), DL 2 (dot), and DL 3 (triangle). Lines are from the CREME model. lower panel: Ratio of the abundance ratio of CREME96 and HIT

We also compared the area passive dosimeter for life science experiments in space (Area PADLES). ${ }^{8)}$ The dosimeter is inside the ISS Kibo, and LET fluxes are obtained every six months. PADLES is a compact and battery-absent dosimeter with a solid-state track detector (CR-39) and thermoluminescent dosimeters (TLD-MSO). TLDs measure the absorbed space radiation doses in the LET regions below $10 \mathrm{keV} / \mu \mathrm{m}$, whereas the CR-39 measures the LET distributions for heavy ions in the LET regions above $10 \mathrm{keV} / \mu \mathrm{m}$. Seventeen PADLES were installed on the surfaces of the interior walls of Kibo with average shielding thickness of $22 \mathrm{~g} / \mathrm{cm}^{2}$. Radiation data have been collected since Kibo was attached to the ISS in 2008. Figure 14 shows the LET distributions obtained with HIT and those averaged from 17 sets of PADLES from March 2009 to September 2009, shown as AP\#2, and from August 2009 to April 2010, shown as AP\#3. Although both sets of LET fluxes are consistent, the element peaks are not observed in the results from PADLES, probably because of nuclear reactions in the ISS wall and racks. 


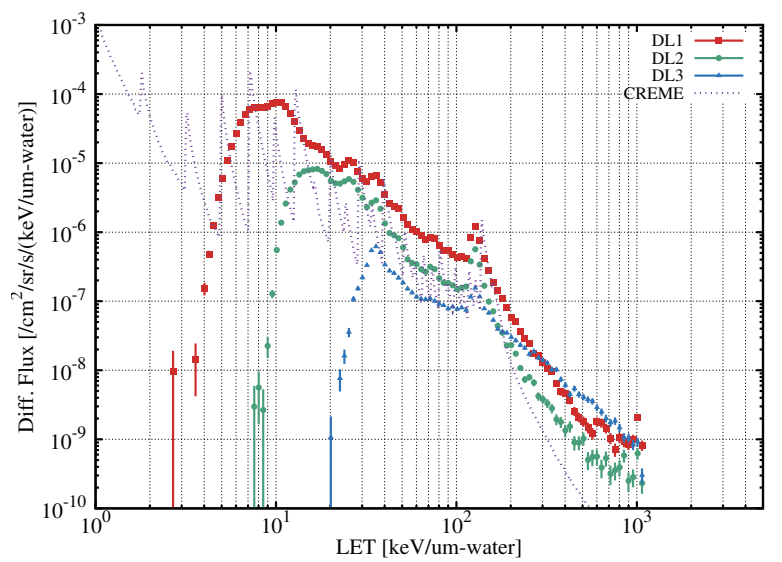

Fig. 13. Differential LET flux distribution for each DL. Squares, dots, triangles, and lines are for DL1, DL2, DL3, and the CREME96 model, respectively.

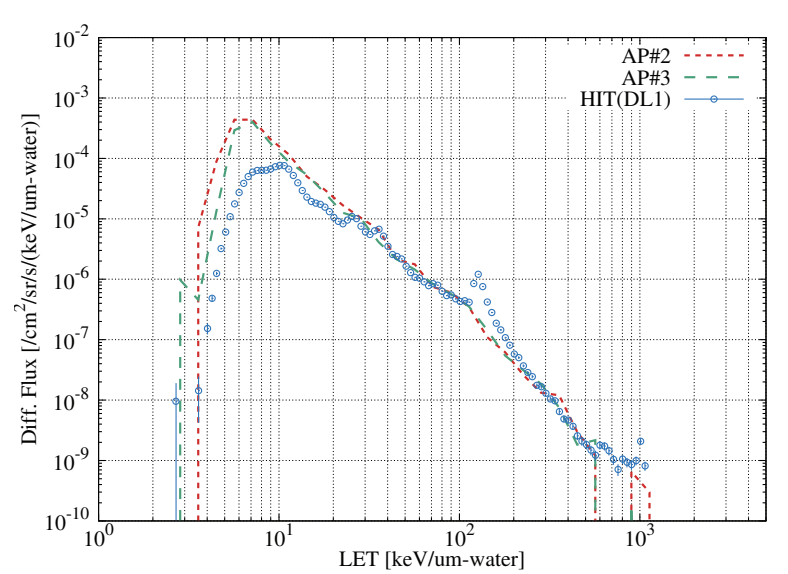

Fig. 14. LET distributions obtained with HIT (open circle) and area PADLES (lines).

\section{Discussion}

\subsection{Solar flares contributions}

Different trends appeared in the particle identification and differential LET flux distributions in the DL3 data. The results suggest that HIT observed heavy ions from solar flares. The largest solar flare in the DL3 data $(2010$ - 2012) was a X5.4 class flare, which occurred on 2012/03/07 at 00:02:00. The flare coordinates were N18 E31 and the coronal mass ejection (CME) was emitted toward Earth. Figure 15 shows the x-ray and proton fluxes of the GOES satellite and HIT count rates vs time. The HIT count rates increased to $\sim 300$ counts/min simultaneously with the increase in the proton flux. Figure 16 represents the comparison of data in the increasing time of count rate and model for $6 \leqslant z \leqslant 16$. For comparison, solar abundance values are taken from Grevesse and Anders, ${ }^{9)}$ except for the solar iron abundance $\left.(\ln (\mathrm{Si})=6) .{ }^{10}\right)$ It can be seen that the flareonly data are closer to the solar abundance than the CREME model for $6 \leqslant z \leqslant 16$. This suggests that heavy ions are possibly observed from this solar flare. Whereas higher $z(z \sim 26)$ ions from this solar flare are not detected by HIT. These data may provide a hint for acceleration mechanism for solar flare.

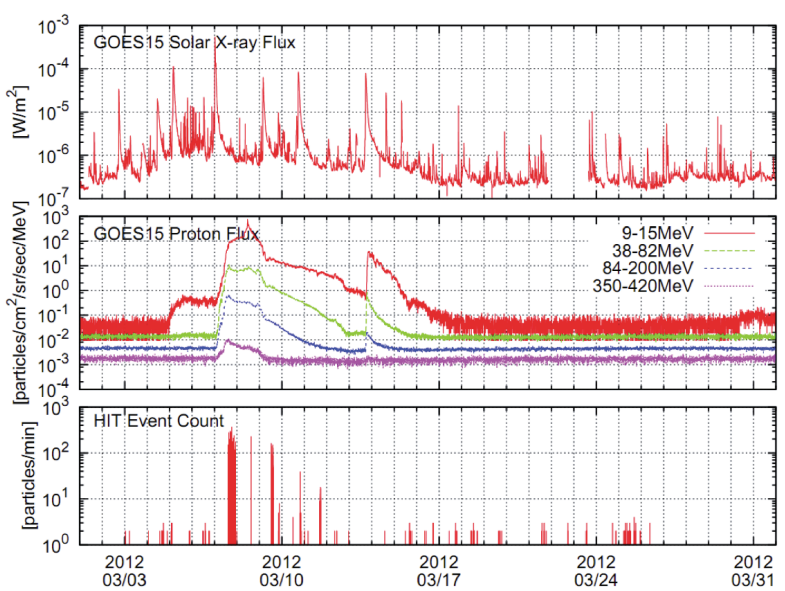

Fig. 15. Time evolution of $\mathrm{x}$-ray (top) and proton (middle) fluxes from GOES and count rates of HIT (bottom).

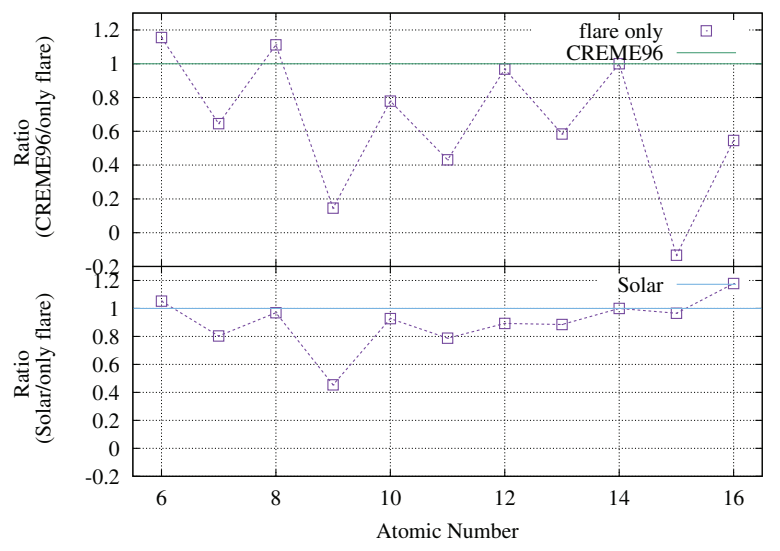

Fig. 16. Ratio of the abundance ratio between the flare-only data (open square) and CREME model (upper panel), and solar abundance (lower panel). The abundance ratio is normalized by $\ln (\mathrm{Si})$.

\subsection{The possibility of observing ACR events}

ACR, which are believed to derive from neutral elements surrounding the solar system, show features that differ from those of GCR or SEP. They are thought to originate from the ionization of neutral interstellar matter, accelerated at the termination of the solar system and returning to earth. ACRs have high first ionization energies, detected as $\mathrm{N}$ and $\mathrm{O}$. Monovalent ionized ACR can intrude into low altitudes because the rigidity of the geomagnetic field is high. Blake et al. ${ }^{11)}$ focused on this point and suggested that ACR at low altitudes are ionized by the upper atmosphere, and then, the rigidity drops sharply; thus, geomagnetic fields trap ACR to form a radiation belt.

The MIDORI satellite measured heavy ions with a HIT at 800 $\mathrm{km}$ altitude from 1996 to $1997 .{ }^{12}$ ) The MIDORI HIT, which had approximately the same structure as the SEDA-AP HIT, observed geomagnetically trapped $\mathrm{N}, \mathrm{O}$, and $\mathrm{Mg}$ near $\mathrm{L}$ value $=$ 2. According to the distributions of the detected ions in section 4.1 , it seems that the SEDA-AP HIT did not observe the heavyion radiation belt even though, in the case of $\mathrm{N}$ and $\mathrm{C}$, several events were observed near the radiation belt. It is likely that the heavy-ion radiation belt was not detected because it was above the approximately $400 \mathrm{~km}$ altitude of the ISS or the ACR pitch angle was out of the field of view of the HIT. 


\section{Conclusion}

For five and a half years, HIT detected more than 700,000 events, identifying the elements in $1.4 \%$ of these events. The elemental abundances and LET distributions were almost consistent with the CREME96 model. The LET fluxes in HIT and PADLES measurements were also consistent. Therefore, we conclude that HIT observations are accurate. HIT may have detected heavy ions from a X5.4 solar flare, which occurred on March 7, 2012. In terms of the possibility of observing ACR, HIT did not detect ACR because it was probably distributed at altitudes higher than $400 \mathrm{~km}$ or the pitch angle of the ACR was out of the field of view of HIT.

\section{Acknowledgments}

We wish to thank Mr. Fujii for his advice and for the processing program for particle identification.

\section{References}

1) Von Rosenvinge, T. T., Barbier, L. M., Karsch, J., Liberman, R., Madden, M. P., Nolan, T., Reames, D. V., Ryan, L., Singh, S., Trexel, H., Winkert, G., Mason, G. M., Hamilton, D. C., Walpole, P.: The energetic particles: Acceleration, composition, and transport (EPACT) investigation on the Wind spacecraft. Space Science Reviews, 71(1995), pp. 155-206.

2) Koga, K., Matsumoto, H., Obara, T., Kimoto, Y., Yamada, N., Watanabe, H., Endo, M., Sakoh, D., Matsueda, T., Yamamoto, T., and Muraki, Y.: Initial Result of Space Environment Data Acquisition Equipment-Attached Payload (SEDA-AP) on the ISS - Kibo Exposed Facility, Proc, RASEDA-9(2010).

3) Goka, T., \& Matsumoto, H.: High Energy Particle Environment. Review of the Communications Research Laboratory, 43(1997), pp. 249, 43, 249.

4) Sato, T., Niita, K., Matsuda, N., Hashimoto, S., Iwamoto, Y., Noda, S., Ogawa, T., Iwase,H., Nakashima,H., Fukahori, T., Okumura, K., Kai, T., Chiba, S., Furuta, T. and Sihver, L.: Particle and heavy ion transport code system, PHITS. Journal of Nuclear Science and Technology, 50(2013), pp. 913-923.

5) Tylka, A. J., Adams Jr, J. H., Boberg, P. R., Brownstein, B., Dietrich, W. F., Flueckiger, E. O., Flueckiger, E. L. Petersen, M. A. Shea, D. F. Smart, Smith, E. C.: CREME96: A revision of the cosmic ray effects on micro-electronics code. Nuclear Science, IEEE Transactions on, 44(1997), pp. 2150-2160

6) Weller, R., Mendenhall, M. H., Reed, R., Schrimpf, R. D., Warren, K. M., Sierawski, B. D., \& Massengill, L. W.: Monte Carlo simulation of single event effects. Nuclear Science, IEEE Transactions on, 57(2010), pp. 1726-1746.

7) Mendenhall, M. H., \& Weller, R. A.. A probability-conserving crosssection biasing mechanism for variance reduction in Monte Carlo particle transport calculations. Nuclear Instruments and Methods in Physics Research Section A: Accelerators, Spectrometers, Detectors and Associated Equipment, 667(2012), pp. 38-43.

8) Nagamatsu, A., Murakami, K., Kitajo, K., Shimada, K., Kumagai, H., \& Tawara, H.: Area radiation monitoring on ISS Increments 17 to 22 using PADLES in the Japanese Experiment Module Kibo. Radiation Measurements, 59(2013), pp. 84-93.

9) N. Grevesse and E. Anders (1988) in Cosmic Abundances of Matter (ed. J. Waddington), Amer. Inst. Phys., New York, p. 1.

10) Holweger, H., Bard, A., Kock, M., \& Kock, A.. A redetermination of the solar iron abundance based on new Fe I oscillator strengths. Astronomy and Astrophysics, 249(1991), pp. 545-549.

11) Blake, J. B., \& Friesen, L. M.:. A technique to determine the charge state of the anomalous low-energy cosmic rays. In International Cosmic Ray Conference, 2(1977), pp. 341-346.
12) Kohno, T., Miyasaka, H., Yamagiwa, I., Kato, C., Goka, T., \& Matsumoto, H.: Heavy ion observation with MIDORI satellite: trapped ACR. Radiation Measurements, 30(1999), pp. 639-644. 